\title{
Los estados contables de la gestión financiera en las empresas de consumo masivo
}

\author{
The financial statements of financial management in mass consumption companies
}

\begin{tabular}{l|r|}
\cline { 2 - 2 } & Deyanira Arguello \\
\hline $\begin{array}{l}\text { Artículo recibido en mayo } 2019 \\
\text { Arbitrado en junio 2019 } \\
\text { Publicado en septiembre } 2019\end{array}$ & lanana2403@hotmail.com \\
& ORCID: 0000-0002-0230-8995 \\
Universidad del Zulia - Venezuela
\end{tabular}

RESUMEN

Palabras clave:

\begin{abstract}
\end{abstract}
Keywords:
El objetivo se enmarcó en determinar los estados contables de la gestión financiera aplicados en las empresas de consumo masivo. Metodológicamente la investigación se contextualizó dentro de un tipo descriptiva; apoyada en un diseño no experimental, transeccional de campo. La población estuvo conformada por los gerentes, subgerentes, coordinadores de ventas, y supervisores de departamento, de las empresas de consumo masivo ubicadas en el Municipio Cabimas, a quienes se les aplico un cuestionario, conformado por 9 ítems. La validez se hizo bajo el juicio de expertos. Para la confiabilidad, se empleó el método Alfa de Cronbach, obteniendo un resultado de 0,83. El análisis de los datos se realizó mediante el análisis de frecuencias absolutas y relativas. Se observó, que solo los estados de situación económica y patrimonial se catalogaron con muy alta aplicación, mientras que los estados de rentabilidad y de circulación financiera se denotaron con alta aplicación.

De circulación financiera, de rentabilidad, de situación económica y patrimonial, estados contables, gestión financiera

The objective was framed in determining the financial management accounting statements applied in mass consumption companies. Methodologically the research was contextualized within a descriptive type; supported by a non-experimental, transectional field design. The population was made up of the managers, assistant managers, sales coordinators, and department supervisors of the mass consumption companies located in the Cabimas Municipality, to whom a questionnaire was applied, made up of 9 items. Validity was made under expert judgment. For reliability, the Cronbach's Alpha method was used, obtaining a result of 0.83 . Data analysis was performed by analyzing absolute and relative frequencies. It was observed that only the statements of economic and patrimonial situation were cataloged with very high application, while the statements of profitability and financial circulation were denoted with high application.

Financial circulation, profitability, economic and patrimonial situation, financial statements, financial management 


\section{INTRODUCCIÓN}

En el amplio mundo de empresas de consumo masivo existe una enorme variedad que puede ir desde la fabricación y distribución del producto más sencillo y pequeño como un alfiler o un fosforo, hasta la elaboración y distribución del más avanzado y complicado como son los alimentos, por lo tanto se puede identificar que hablar de empresas de consumo masivo es un amplio universo en el cual se encuentran empresas que elaboran $y$ distribuyen sus propios productos, solo fabrican, y otras se dedican exclusivamente a distribuir los que otros producen.

Dentro de estas clasificaciones, también se pueden encontrar empresas de todo tipo de tamaño y cobertura geográfica, las cuales tratan de satisfacer las diferentes necesidades de la población a nivel regional sin importar el nivel social al que pertenezcan los consumidores, utilizando métodos de distribución basada en una logística bien planificada y especifica de acuerdo a la especialidad y aplicaciones de los productos.

Al respecto, Vera (2001), afirma que en Venezuela, los inicios de las empresas de consumo masivo se remonta a la década de los años 30 posterior a la segunda guerra mundial, en donde comenzó a generar una mayor demanda de productos de usos diario de los hogares y se presentó la necesidad de abastecer y facilitar esta amplia variedad de artículos en un tiempo menor comenzando una etapa nueva comercial.

Así las cosas, empresas de consumo masivo venezolanas y trasnacionales han incursionado en la región occidental del país, así como lo han venido haciendo en otras regiones, estableciéndose en ciudades según las necesidades de la población. Esta situación, también está presente en el municipio Cabimas, donde las empresas de consumo masivo se han desarrollado progresivamente con el propósito de expandir y segmentar el mercado de la zona, producto de la creciente competencia y las nuevas condiciones del mercado que llevan a replantearse el esquema de mejora de los resultados basado en una desaceleración importante del aumento de los precios de ventas.

En este contexto los gerentes están conscientes del papel determinante que ejerce la gestión financiera como fuerza impulsora de la actividad económica de toda organización. Al respecto, Ortiz (2005), afirma que la gestión financiera induce a las organizaciones asumir condiciones de categoría súper paradigmáticas, la cual se sobrepone a otros aspectos importantes de la administración financiera: liderazgo, estrategia, tecnología y estados contable, considerando a partir de su estructura presentar soluciones a los problemas organizacionales, destacando la importancia de la gestión, enfatizando procesos de mejora continua con el compromiso de sus funcionarios, cumpliendo los objetivos establecidos, y señalando las deficiencias entre empresas.

Por otra parte, los estados contables constituyen la base de información para el analista a la hora de desarrollar su trabajo. Su importancia radica en que permite identificar los aspectos económicos y financieros que muestran las condiciones en que opera la empresa con respecto al nivel de liquidez, solvencia, endeudamiento, eficiencia, rendimiento y rentabilidad, facilitando la toma de decisiones gerenciales, económicas y financieras en la actividad empresarial. 
Frente a esta realidad la investigadora reflexiona y observa las estrategias a nivel de finanzas de las empresas de consumo masivo ubicadas en el municipio Cabimas, evidenciándose que estas empresas se caracterizan por ser netamente financieras debido a que su actividad económica que es comercializar productos de alimentos y hogar entre otros, para satisfacer las necesidades de los consumidores, principalmente son negociados con los proveedores quien les distribuye los artículos financiados a crédito y estos los venden de contados para obtener margen de rentabilidad deseados a cada rubro comercializado.

En este sentido y en virtud de la problemática aquí expuesta, se formula la siguiente interrogante: ¿Cuáles son los estados contables de la gestión financiera aplicados en las empresas de consumo masivo del municipio Cabimas?

\section{Aspectos teóricos}

\section{Gestión financiera}

Se denomina gestión financiera, según Sabino (2007), a todos los procesos que consisten en conseguir, mantener y utilizar dinero, sea físico (billetes y monedas) o a través de otros instrumentos, como cheques y tarjetas de crédito. La gestión financiera es la que convierte a la visión y misión en operaciones monetarias. Afirma este autor que, la gestión financiera es una de las tradicionales áreas funcionales de la gestión, hallada en cualquier organización, competiéndole los análisis, decisiones y acciones relacionadas con medios financieros necesarios a la actividad de dicha organización. Así, la función financiera integra todas las tareas relacionadas con el logro, utilización y control de recursos financieros.

En este marco referencial, para Gitman (2007) la gestión financiera trata lo concerniente a la implementación de acciones políticas tendientes a lograr la eficiente administración de los recursos económicos, asignados o generados por la organización. Teniendo como objetivo influir en el comportamiento de los costos e ingresos, a fin de disponer de los suficientes recursos, para llevar a cabo la ejecución de los procesos productivos.

De igual manera, acita este autor, es la responsable de proveer todas las herramientas para realizar las operaciones fundamentales de la empresa frente al control de las inversiones, el manejo de recursos, entre otros, en adquisición a las nuevas fuentes de financiamiento, eficiencia operacional, administrativa, de alta confiabilidad en la información financiera, suministrada a el cumplimiento de las leyes, aplicables a las regulaciones estipuladas al momento.

Al respecto, Ruiz (2005) afirma que la gestión financiera está relacionada con la toma de decisiones relativas al tamaño y composición de los activos, al nivel y estructura de la financiación y a la política de los dividendos. Se puede decir que a fin de tomar las decisiones adecuadas es necesaria una clara comprensión de los objetivos que se pretenden alcanzar, debido a que el objetivo facilita un marco para una óptima toma de decisiones financieras.

Con base en lo expuesto, la investigadora considera que, la gestión financiera abarca el conjunto operativo de compra - venta, instrumentos legales, cuyos propietarios tiene ciertos derechos para 
percibir en el futuro una determinada cantidad monetaria. Estos instrumentos legales se denominan activos financieros o títulos de valores, incluyendo bonos, acciones, préstamos otorgados por instituciones financieras, entre otros.

\section{Estados contables de la gestión financiera}

Los estados financieros, también denominados estados contables, informes financieros o cuentas anuales, son informes que utilizan las instituciones para dar a conocer la situación económica financiera y los cambios que experimenta la misma a una fecha o periodo determinado, según lo afirma Castán (2005). Esta información resulta útil para la administración o gestión, gestores, reguladores y otros tipos de interesados como los accionistas, acreedores o propietarios.

En esta perspectiva, según Da Silva (2007) el objetivo de los estados financieros es proveer información sobre el patrimonio del emisor a una fecha, y su evolución económica y financiera en el período que abarcan, para facilitar la toma de decisiones. Ahora bien, de acuerdo con Koontz y Weihrich (2005) los estados financieros presentan la salud financiera de un organismo o de una empresa. Son documentos en los que se resume el conjunto de los datos financieros registrados en el sistema contable para un período determinado. Sus datos se presentan de manera organizada con el fin de facilitar la toma de decisiones de los distintos usuarios.

De acuerdo con los autores anteriores se dice que, los informes financieros deben estar disponibles a la brevedad suficiente para que puedan ser útiles en la solución de los problemas de la empresa y poder valorar el rendimiento de las diferentes dependencias de la entidad con el objetivo de tomar decisiones adecuadas en el momento oportuno. Los términos empleados para describir la información financiera y presentación material en los estados deberán ser muy claras, con el objetivo de facilitar su compresión ante los usuarios, deben omitirse los extremos de excesiva brevedad o demasiado detalles.

En finanzas, dice Escribano (2011), los informes financieros son la característica por la cual el usuario acepta y utiliza la información contable para formar decisiones con base en ella, estableciendo una estrecha relación entre el usuario y la información financiera suministrada. Dicha información debe ser comprobable debido a que permite que los estados financieros puedan ser revisados posteriormente y puedan aplicarse para comprobar la información producida, ya que son explicitas sus reglas de operación.

A su vez, continúa el autor, implica que la información financiera contenida en los estados ha sido presentada de acuerdo a la realidad, y que las reglas del sistema no han sido distorsionadas. La no distorsión significa que no presenta hechos totalmente acabados ni terminados. Cierra la idea afirmando que, los informes financieros son el producto de la necesidad de hacer cortes en la vida de la empresa, para presentar los resultados de las operaciones y la situación financiera y sus cambios incluyendo eventos cuyos efectos no terminan a la fecha de cierre de los estados financieros.

A efectos de esta investigación, se entenderá por estados contables de la gestión financiera el producto final de la contabilidad de una organización o empresa, elaborados de acuerdo a principios de contabilidad generalmente aceptados, 
normas contables o normas de información financiera. Citando a Castán (2005), los estados financieros obligatorios dependen de cada país, siendo los componentes más habituales los siguientes:

$\checkmark$ Estado de situación patrimonial (también denominado estado de situación financiera, balance general o balance de situación).

$\checkmark$ Estado de resultados (también denominado estado de pérdidas $y$ ganancias o cuenta de pérdidas y ganancias).

$\checkmark$ Estado de evolución de patrimonio neto (también denominado estado de cambios en el patrimonio neto).

$\checkmark$ Estado de flujo de efectivo

$\checkmark$ Memoria

Así las cosas, la investigadora consideró conveniente asumir como indicadores de esta dimensión los estados contables siguientes: de situación económica y patrimonial, de rentabilidad y de circulación financiera; por considerarlos los más pertinentes en las empresas bajo estudio.

\section{De situación económica y patrimonial}

El activo incluye todas aquellas cuentas que reflejan los valores de lo que dispone la entidad, por el contrario, el pasivo: muestra todas las obligaciones ciertas ante el ente y contingencias que deben registrarse (Escribano, 2011). Afirma que, estas obligaciones son, naturalmente, económicas: préstamos, compras con pago diferido, entre otros. La situación económica se refiere a lo patrimonial. Se tiene buena situación económica cuando el patrimonio es importante. El patrimonio es la diferencia entre activo y pasivo.
Bajo esta perspectiva, para Andrade (2005) la situación financiera patrimonial se refiere a la liquidez que se tiene cuando se cuenta con abundantes medios de pago, producto de una adecuada gestión financiera, que conlleva a determinar una buena o mala situación económica. Por ejemplo, si se obtiene un préstamo o crédito y se cuenta con gran cantidad de efectivo o bienes asimilables a efectivo pero las deudas superan a los activos. Afirma este autor que, puede tenerse buena situación económica y mala situación financiera; por ejemplo, cuando se cuenta con muchos activos pero el efectivo y bienes similares resultan insuficientes para hacer frente a las deudas.

Es importante destacar que, según Sabino (2007), dentro de una adecuada gestión financiera se debe contar con un balance, definido como el estado contable que proporciona información sobre la situación económica y financiera de la empresa en un momento o tiempo determinado. Está formado por el activo, pasivo y patrimonio neto: el activo refleja lo que se tiene, empleo de fondos (inversiones en el local, en maquinaria, valor del almacén, saldos pendientes de cobro de nuestros clientes, saldo de tesorería), representa las inversiones de la empresa, el capital en funcionamiento y el destino de los recursos. Podría decirse que el activo contabiliza lo que la empresa posee. Pasivo y patrimonio neto reflejan lo que se debe y a quién se le debe, es el origen de fondos.

Vistas las definiciones expuestas, la investigadora deduce que el estado de situación económica y patrimonial permite exponer, de acuerdo con la legislación vigente, el resultado estático de la situación patrimonial, económica y financiera de un 
ente, a un momento determinado. Para ello, se conserva la estructura de la ecuación patrimonial estática: activo $=$ pasivo + patrimonio neto, e incluye en cada uno de estos diferentes rubros que agrupan las cuentas que fueron utilizadas en el ejercicio económico. A fines de la gestión financiera, este estado permite evaluar el grado de endeudamiento de la empresa y su equilibrio financiero; asimismo, refleja la garantía que la empresa ofrece a sus prestamistas, proveedores, acreedores y terceras personas; así como conocer el grado de liquidez para solventar las obligaciones inmediatas.

\section{De rentabilidad}

La rentabilidad, a juicio de Escribano (2011), es pieza clave en el manejo de la administración financiera, y da respuesta a preguntas de que tan efectiva está la empresa, a su vez mide la efectividad de los rendimientos generados de las ventas y sobre las inversiones de la empresa. Este principio es muy importante en los estados contables de la gestión financiera ya que mide el rendimiento sobre el capital invertido.

En esta línea de pensamiento, para Samuelson y Nordhaus (2005) la rentabilidad financiera o «ROE» relaciona el beneficio económico con los recursos necesarios para obtener ese lucro. Dentro de una empresa, muestra el retorno para los accionistas de la misma, que son los únicos proveedores de capital que no tienen ingresos fijos. La rentabilidad puede verse como una medida de cómo una compañía invierte fondos para generar ingresos. Se suele expresar como porcentaje.

Así las cosas, la rentabilidad de la empresa es uno de los parámetros que afectan a la eficiencia, según lo afirman Skousen y Addison (2007). La consecución de este objetivo supone efectuar un análisis de la cuenta de pérdidas $y$ ganancias. Explican los autores que, metodológicamente, se estudian los componentes de la cuenta de resultados, a través de índices verticales y horizontales, así como diferentes ratios. El margen de rentabilidad es la ganancia obtenida en relación al monto de la venta.

Al detalle, si se venden 100 y la ganancia es 10 , el margen de rentabilidad será del $10 \%$. Señalan que, a veces, los empresarios no conocen el monto de la venta y solo cuentan con el monto del costo, entonces aplican el porcentaje deseado a este costo, obteniendo el margen por debajo de lo planeado, lo cual es un error. Cabe destacar que los ratios financieros de rentabilidad se establecen en relación a la venta y no al costo.

La rentabilidad, según Andrade (2005), da respuestas más completas a la pregunta de qué tan efectivamente está siendo manejada la empresa. Mide la efectividad de la administración a través de los rendimientos generados sobre las ventas y sobre las inversiones. Proporciona algunas claves útiles en la gestión financiera en cuanto a la forma en la que se está operando la empresa.

Dice este autor que, una empresa es rentable cuando genera suficiente utilidad o beneficio, es decir, cuando sus ingresos son mayores a sus gastos, y la diferencia entre ellos es considerada como aceptable, pero lo correcto al momento de evaluar la rentabilidad de una empresa es evaluar la relación que existe entre sus utilidades 0 beneficios, y la inversión o los recursos que 
ha utilizado para obtenerlos, es por ello la importancia de los estados financieros.

En este punto, para la investigadora la rentabilidad económica o del activo consiste en analizar la rentabilidad del activo independientemente de cómo está financiado el mismo, o dicho de otra forma, sin tener en cuenta la estructura del pasivo. Su aplicabilidad en la gestión financiera viene dada porque proporciona información clave en cuanto a la forma en la que se está operando la empresa, midiendo la efectividad de la gestión a través de los rendimientos generados sobre las ventas y las inversiones.

\section{De circulación financiera}

Consiste en explicar cómo se analiza un estado de circulación financiera, ya sea un cuadro de financiación o un estado de flujo de efectivo (Escribano, 2011). Al respecto, para Skousen y Addison (2007), el análisis de un estado de circulación financiera se basa en el estudio de la cuantía y procedencia de las fuentes de financiación captadas en el ejercicio así como de su empleo o aplicación. El análisis resulta de especial utilidad cuando se clasifican los flujos por actividades: flujos de fondos de explotación o por operaciones corrientes, flujos de fondos por actividades de financiación, flujos de fondos por actividades de inversión.

En este marco referencial, la circulación financiera básica se desarrolla entre empresas, mercados, individuos y estado; el mismo destaca que una empresa es una unidad de intercambio de dinero y recursos que involucra en última instancia a los individuos (clientes, proveedores, personal, propietarios, prestamistas), según lo afirmado por Fornero (2007). Asimismo este autor considera que, el dinero para financiar los activos de la empresa se obtiene de los individuos, directamente 0 con intermediarios en los mercados financieros, y la existencia de mercados financieros facilita las transacciones con las que, finalmente, se financian las empresas.

En este orden de ideas, para Andrade (2005), las operaciones con esos activos (que involucran transacciones con otras empresas y con individuos) implican cobros y pagos, y generan un excedente, el cual se destina a nuevas inversiones en activos, a intereses de las deudas y dividendos de los propietarios, a pago de las deudas y a impuestos. Por ello, los individuos cierran la circulación financiera, puesto que, son los individuos los que reciben y entregan el dinero, como retribuciones por trabajo, pagos de productos y servicios, aportes de capital, préstamos, dividendos, intereses, impuestos.

Afirma el autor citado que, en el circuito financiero de una economía existe una gran cantidad de etapas en las que intervienen instituciones (empresas de negocios, intermediarios financieros, Estado), y que son proveedores y clientes unas de otras. Los individuos de esas organizaciones, a través del trabajo directivo o el trabajo operativo, hacen que funcione el sistema. Por tal motivo, queda claro que los consumidores y los poseedores de capital (financiero y humano) son, en última instancia, los individuos. Las personas individuales, en unidades familiares, y no las instituciones.

La circulación financiera, vista por la investigadora, implica ciclos sucesivos de dinero a bienes, y de bienes a dinero entre empresas e individuos. Las operaciones de un negocio se manifiestan en dos tipos de 
ciclos financieros, que difieren en su ritmo. El ciclo financiero corto resulta del hecho de que se destinan fondos para insumos o productos, dinero que se recupera con el cobro de las ventas. El ciclo financiero largo es el de los recursos fijos o activo fijo (marcas, equipamiento, intangibles, entre otros).

Los fondos destinados a esos recursos retornan como dinero en sucesivos períodos, a través de las ventas que se realizan utilizando esa capacidad. Entonces, a diferencia de los fondos que se destinan a insumos, que se recuperan con la venta de los productos, la recuperación de las inversiones fijas no se produce principalmente con la venta de esos activos, sino a través de la generación operativa de fondos que resulta del uso del activo fijo.

MATERIALES Y METODO

Metodológicamente la investigación se contextualizó dentro de un tipo descriptiva; apoyada en un diseño no experimental, transeccional de campo. La población estuvo conformada por los gerentes, subgerentes, coordinadores de ventas, y supervisores de departamento, de las empresas de consumo masivo ubicadas en el Municipio Cabimas. Se trata pues, de una población finita y accesible a la posibilidad de la investigadora, por lo que se prescindió de la técnica del muestreo y se empleó el censo poblacional.

De acuerdo al enfoque elegido, en este caso de estudio, la técnica que se utilizó para la recolección de la información fue la encuesta, y como instrumento el cuestionario con escala de respuesta de frecuencia, conformado por 9 ítems. La validez se hizo bajo el juicio de expertos en el área específica, tres (03) en contenido y dos (02) en metodología. Para la confiabilidad, se empleó el método Alfa de Cronbach, obteniendo un resultado de 0,83 . El análisis de los datos se realizó mediante las frecuencias absolutas y relativas. A tal efecto, la investigadora consideró conveniente fijar un baremo de interpretación el cual se recoge en el cuadro 1.

Tabla 1. Interpretación del porcentaje de la distribución de frecuencia

\begin{tabular}{ccc}
\hline Rango & Categoría & Interpretación \\
\hline $75 \% \leq S+C S<100 \%$ & $\begin{array}{c}\text { Muy alta aplicación } \\
\text { Alta fortaleza }\end{array}$ & Muy alta aplicación e implica alta fortaleza \\
$50 \% \leq S+C S<75 \%$ & $\begin{array}{c}\text { Alta aplicación } \\
\text { Leve fortaleza }\end{array}$ & Alta aplicación e implica leve fortaleza \\
$25 \% \leq S+C S<50 \%$ & $\begin{array}{c}\text { Baja aplicación } \\
\text { Leve debilidad } \\
\text { Muy baja aplicación } \\
\text { Alta debilidad }\end{array}$ & Baja aplicación e implica leve debilidad \\
\hline $5 \leq S+C S<25 \%$ & Muy baja aplicación e implica alta debilidad \\
\hline
\end{tabular}

Fuente: Arguello (2020) 
RESULTADOS Y DISCUSION

En lo que respecta al objetivo dirigido a determinar los estados contables de la gestión financiera aplicados en las empresas de consumo masivo del municipio Cabimas, el cual dio origen a la dimensión denominada estados contables de la gestión financiera, cuyos indicadores son: de situación económica y patrimonial, de rentabilidad y de circulación financiera, se presentan los siguientes resultados:

Se muestra la tabla 2, la cual recoge los hallazgos obtenidos para el indicador estado de situación económica y patrimonial, donde se aprecia que para el $77,78 \%$ de los encuestados, en promedio, opinan que el estado de situación económica y patrimonial como parte de los estados contables de la gestión financiera posee muy alta aplicación en las empresas bajo estudio, confiriéndoles a éstas una alta fortaleza al respecto.

Estos resultados obedecen a las categorías dada a los ítems, donde se evidencia que un $77,78 \%$ de los encuestados opina que, siempre y casi siempre, elaboran estado de evolución del patrimonio neto, la actividad productiva va de la mano con el estado de ganancias y pérdidas y elaboran un estado contable anual que propicie información sobre la situación económicafinanciera, confiriéndoles a estas actividades muy alta aplicación convirtiéndolas en altas fortalezas.

Tabla 2. Indicador: De situación económica y patrimonial

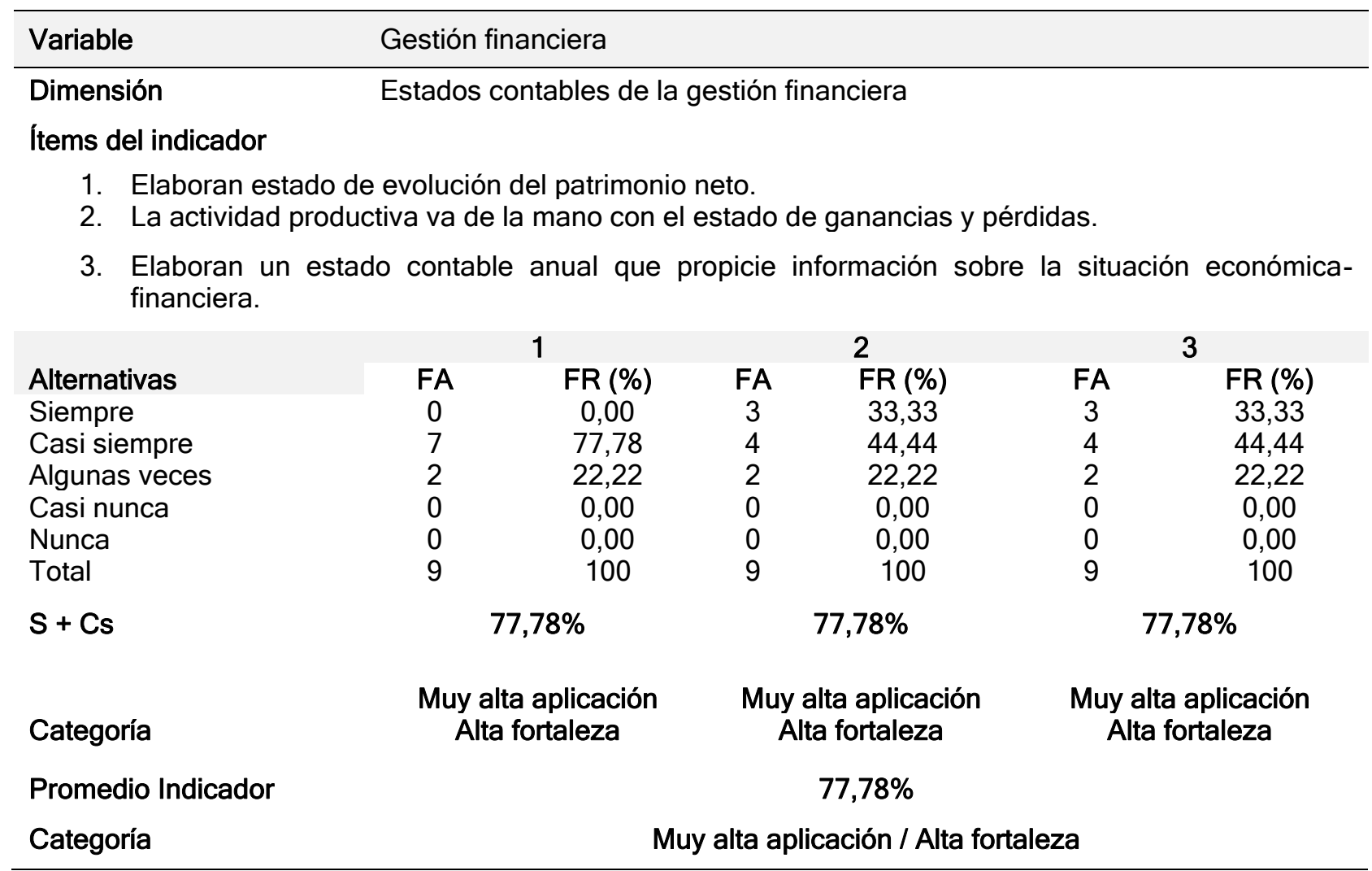

Fuente: Arguello (2020) 
Los resultados muestran muy alta congruencia con lo expuesto por Sabino (2007), para quien dentro de una adecuada gestión financiera se debe contar con un balance, definido como el estado contable de situación económica y patrimonial que proporciona información sobre la situación económica y financiera de la empresa en un momento o tiempo determinado.

Visto así, para la investigadora, las empresas analizadas han logrado dar el alto valor a este estado contable, el cual les permite evaluar el grado de endeudamiento de la empresa y su equilibrio financiero; asimismo, la garantía que la empresa ofrece a sus prestamistas, proveedores, acreedores y terceras personas; así como conocer el grado de liquidez para solventar las obligaciones inmediatas.

En lo concerniente al indicador estado contable de rentabilidad, se observa, en la tabla 3, un promedio de frecuencias de $66,67 \%$, indicando alta aplicación de este indicador como estado contable de la gestión financiera en las empresas analizadas, implicando una leve fortaleza, según el baremo diseñado. Así, en el detalle de los ítems, el instrumento diseñado arrojó que el $77,78 \%$ de los encuestados señalaron que, siempre y casi siempre, se le da muy alta aplicación al que le den a conocer a todo el personal el margen de intereses generales y midan el beneficio después del pago de impuestos, otorgándole altas fortalezas a las empresas bajo estudio, en lo que respecta a estos aspectos. Mientras que los encuestados se posicionaron en la categoría de baja aplicación (44,44\%), al consultarles si consideran el margen utilidad en relación al capital invertido, denotando este aspecto como una leve debilidad.

Tabla 3. Indicador: De rentabilidad

\begin{tabular}{|c|c|c|c|c|c|}
\hline Variable & \multicolumn{5}{|l|}{ Gestión financiera } \\
\hline Ítems del indicador & \multicolumn{5}{|c|}{ Estados contables de la gestión financiera } \\
\hline \multicolumn{6}{|c|}{$\begin{array}{l}\text { 4. Le dan a conocer a todo el personal el margen de intereses generales. } \\
\text { 5. Consideran el margen utilidad en relación al capital invertido. } \\
\text { 6. Se mide el beneficio después del pago de impuestos. }\end{array}$} \\
\hline & 4 & \multicolumn{2}{|c|}{5} & \multicolumn{2}{|c|}{6} \\
\hline Alternativas & FR (\%) & FA & FR (\%) & FA & FR (\%) \\
\hline Siempre & 0,00 & 2 & 22,22 & 0 & 0,00 \\
\hline Casi siempre & 77,78 & 2 & 22,22 & 7 & 77,78 \\
\hline Algunas veces & 22,22 & 5 & 55,56 & 2 & 22,22 \\
\hline Casi nunca & 0,00 & 0 & 0,00 & 0 & 0,00 \\
\hline Nunca & 0,00 & 0 & 0,00 & 0 & 0,00 \\
\hline Total & 100 & 9 & 100 & 9 & 100 \\
\hline$S+C s$ & $77,78 \%$ & \multicolumn{2}{|c|}{$44,44 \%$} & \multicolumn{2}{|c|}{$77,78 \%$} \\
\hline Categoría & $\begin{array}{l}\text { Muy alta aplicación } \\
\text { Alta fortaleza }\end{array}$ & \multicolumn{2}{|c|}{$\begin{array}{l}\text { Baja aplicación } \\
\text { Leve debilidad }\end{array}$} & \multirow{2}{*}{\multicolumn{2}{|c|}{$\begin{array}{l}\text { Muy alta aplicación } \\
\text { Alta fortaleza }\end{array}$}} \\
\hline Promedio Indicador & \multicolumn{3}{|c|}{$66,67 \%$} & & \\
\hline Categoría & \multicolumn{5}{|c|}{ Alta aplicación / Leve fortaleza } \\
\hline
\end{tabular}

Fuente: Arguello (2020) 
Lo mostrado valida lo postulado por Escribano (2011), para quien la rentabilidad es pieza clave en el manejo de la administración financiera, y da respuesta a preguntas de que tan efectiva está la empresa, a su vez mide la efectividad de los rendimientos generados de las ventas y sobre las inversiones de la empresa. Este principio es muy importante en los estados contables de la gestión financiera ya que mide el rendimiento sobre el capital invertido.

En virtud de los resultados obtenidos, a criterio de la investigadora, la evidencia de alta aplicación del estado contable de rentabilidad, como estado contable de la gestión financiera, corrobora lo expuesto por numerosos autores en el área gerencialfinanciera, quienes señalan que la aplicabilidad de este estado contable en la gestión financiera viene dada porque proporciona información clave en cuanto a la forma en la que se está operando la empresa, midiendo la efectividad de la gestión a través de los rendimientos generados sobre las ventas y las inversiones.

Ahora bien, en el orden de presentación que se lleva adelante, se tienen los resultados del último indicador considerado en la dimensión estados contables de la gestión financiera, referido al estado de circulación financiera. En la tabla 4, se puede observar un promedio de frecuencias en las respuestas positivas ( $\mathrm{S}+\mathrm{Cs}$ ) de $66,67 \%$, indicando alta aplicación de este indicador como estado contable de la gestión financiera aplicado por las empresas bajo estudio, lo cual les concede una leve fortaleza.

Tabla 4. Indicador: De circulación financiera

\begin{tabular}{|c|c|c|c|c|c|c|}
\hline Variable & \multicolumn{6}{|c|}{ Gestión financiera } \\
\hline Dimensión & \multicolumn{6}{|c|}{ Estados contables de la gestión financiera } \\
\hline \multicolumn{7}{|c|}{ Ítems del indicador } \\
\hline \multicolumn{7}{|c|}{ 7. Consideran los flujos de fondos un criterio de operaciones o actividades. } \\
\hline \multicolumn{7}{|c|}{ 8. A menudo amplían el capital social. } \\
\hline \multicolumn{7}{|c|}{ 9. Generan los cobros por ventas o prestacior } \\
\hline \multirow[b]{2}{*}{ Alternativas } & \multicolumn{2}{|c|}{7} & \multicolumn{2}{|c|}{8} & \multicolumn{2}{|c|}{9} \\
\hline & FA & $\mathrm{FR}(\%)$ & FA & FR (\%) & FA & FR (\%) \\
\hline Siempre & 3 & 33,33 & 0 & 0,00 & 7 & 77,78 \\
\hline Casi siempre & 4 & 44,44 & 4 & 44,44 & 0 & 0,00 \\
\hline Algunas veces & 2 & 22,22 & 5 & 55,56 & 2 & 22,22 \\
\hline Casi nunca & 0 & 0,00 & 0 & 0,00 & 0 & 0,00 \\
\hline Nunca & 0 & 0,00 & 0 & 0,00 & 0 & 0,00 \\
\hline Total & 9 & 100 & 9 & 100 & 9 & 100 \\
\hline \multirow[t]{2}{*}{$S+C s$} & \multicolumn{2}{|c|}{$77,78 \%$} & \multicolumn{2}{|c|}{$44,44 \%$} & \multicolumn{2}{|c|}{$77,78 \%$} \\
\hline & \multicolumn{2}{|c|}{ Muy alta aplicación } & \multicolumn{2}{|c|}{ Baja aplicación } & \multirow{3}{*}{\multicolumn{2}{|c|}{$\begin{array}{l}\text { Muy alta aplicación } \\
\text { Alta fortaleza }\end{array}$}} \\
\hline \multirow{2}{*}{$\begin{array}{l}\text { Categoría } \\
\text { Promedio } \\
\text { Indicador }\end{array}$} & & leza & & jilidad & & \\
\hline & \multicolumn{4}{|c|}{$66,67 \%$} & & \\
\hline Categoría & \multicolumn{6}{|c|}{ Alta aplicación / Leve fortaleza } \\
\hline
\end{tabular}

Fuente: Arguello (2020) 
Al desglosar resultados, se evidencia muy alta aplicación de las actividades señaladas por los ítems 7 y 9, donde el $77,78 \%$ de los encuestados afirman que con muy alta frecuencia consideran los flujos de fondos un criterio de operaciones 0 actividades y generan cobros por ventas o prestaciones de servicios registrados durante el ejercicio, lo cual les confiere altas fortalezas a las empresas analizadas en estos aspectos. No obstante, el $44,44 \%$ de los encuestados afirman que a menudo amplían el capital social, lo cual le otorga a esta actividad la categoría de baja aplicación indicando leve debilidad en este aspecto.

Los resultados validan a Skousen y Addison (2007), para quienes el análisis de un estado de circulación financiera se basa en el estudio de la cuantía y procedencia de las fuentes de financiación captadas en el ejercicio así como de su empleo o aplicación. El análisis resulta de especial utilidad cuando se clasifican los flujos por actividades: flujos de fondos de explotación o por operaciones corrientes, flujos de fondos por actividades de financiación, flujos de fondos por actividades de inversión.

Dado los resultados mostrados, la investigadora deduce que en las empresas bajo estudio se le está atribuyendo al estado de circulación financiera el rol que debe tener en la gestión financiera, dado que el mismo es tratado con alta aplicación, considerando que este estado explica cómo se analiza un estado de circulación financiera, ya sea un cuadro de financiación o un estado de flujo de efectivo, implicando ciclos sucesivos de dinero a bienes, y de bienes a dinero entre empresas e individuos.

Luego de analizados los resultados para cada uno de los indicadores que conforman la dimensión estados contables de la gestión financiera, en la tabla 5 se reflejan los resultados alcanzados para dicha dimensión. Se observa que la misma se ubica en la categoría de alta aplicación $(70,37 \%)$ indicando que en las empresas de consumo masivo, la aplicación de los estados contables en la gestión financiera constituye en una leve fortaleza.

Tabla 5. Dimensión: Estados contables de la gestión financiera

\begin{tabular}{lcl}
\hline & Variable: Gestión financiera & \\
\hline INDICADOR & $\mathrm{S}+\mathrm{Cs}$ & CATEGORÍAS \\
De situación económica y patrimonial & $77,78 \%$ & Muy alta aplicación / Alta fortaleza \\
De rentabilidad & $66,67 \%$ & Alta aplicación / Leve fortaleza \\
De circulación financiera & $66,67 \%$ & Alta aplicación / Leve fortaleza \\
\multicolumn{1}{c}{ RESUMEN DIMENSIÓN } & $70,37 \%$ & Alta aplicación / Leve fortaleza
\end{tabular}

Fuente: Arguello (2020) 
Así entonces, se observa que el estado contable de situación económica y patrimonial se posiciona en el rango del baremo $75 \% \leq S+C S<100 \%$ implicando muy alta aplicación, lo cual representa alta fortaleza para las empresas analizadas. Mientras que los estados de rentabilidad y de circulación financiera se denotaron con alta aplicación, constituyendo leves fortalezas de la gestión financiera que se analiza.

Estos resultados son coincidentes con lo manejado por Koontz y Weihrich (2004), para quienes los estados financieros presentan la salud financiera de una empresa. Son documentos en los que se resume el conjunto de los datos financieros registrados en el sistema contable para un período determinado. Sus datos se presentan de manera organizada con el fin de facilitar la toma de decisiones de los distintos usuarios.

De igual manera son congruentes con lo afirmado por Castán (2005), cuando afirma que, los estados financieros o contables son informes que utilizan las instituciones para dar a conocer la situación económicafinanciera y cambios que experimenta la misma a una fecha o periodo determinado. Esta información resulta útil para la gestión, gestores, reguladores y otros interesados como los accionistas, acreedores 0 propietarios.

Visto estos resultados de alta aplicación, a juicio de la investigadora, en las empresas de consumo masivo, los estados contables son considerados de manera eficiente en la ejecución de la gestión financiera que desarrollan, puesto que han sido catalogados con alta aplicación, siendo esta situación una leve fortaleza de la gestión. Así las cosas, han entendido que los estados contables de la gestión financiera debe ser el producto final de la contabilidad de sus empresas, y a su vez, deben ser elaborados de acuerdo a principios de contabilidad generalmente aceptados, normas contables o normas de información financiera.

\section{CONCLUSIONES}

Ahora bien, en cuanto al objetivo, en el cual se determinaron los estados contables de la gestión financiera aplicados en las empresas de consumo masivo del municipio Cabimas, se concluye que existe alta aplicación de dichos estados en la gestión financiera analizada. En este punto se observó, que solo los estados de situación económica y patrimonial se catalogaron con muy alta aplicación, mientras que los estados de rentabilidad y de circulación financiera se denotaron con alta aplicación, constituyendo leves fortalezas de la gestión financiera que se analizó.

De manera más concreta, se tiene que los estados de rentabilidad y de circulación financiera aplicados en la gestión financiera, presentan oportunidades de mejora en una actividad fundamental en cada caso, dado que fueron catalogadas con baja aplicación, por lo cual se considera necesario: garantizar que se aplique el margen utilidad en relación al capital invertido y velar porque se amplíe el capital social activando así los flujos de fondos por actividades de inversión.

\section{REFERENCIAS}

Andrade, S. (2005). Diccionario de economía. Editorial Andrade. GranadaEspaña

Castán, J. (2005). Fundamentos y aplicaciones de la gestión financiera de la empresa. Editorial Pirámide. Madrid. España 
Da Silva, R. (2007). Teoría de la administración. Editorial Thompson Learning. México

Escribano, G. (2011). Gestión financiera. Tercera edición. Ediciones Paraninfo. España

Fornero, R. (2007). Técnicas de finanzas. Editorial Mendoza Sociedad Argentina de Docentes en Administración. Argentina

Gitman, L. (2007). El futuro de los negocios. Editorial Thompson. 11va. Edición. México

Koontz, H. y Weihrich, H. (2005). Administración. Una perspectiva global. 12a. Edición. Editorial McGraw-Hill Interamericana. México

Ortiz, A. (2005); Gerencia financiera y diagnostico estratégico, 2da edición. Editorial McGraw-Hill. Colombia
Ruiz, J. (2005). Fundamentos para el análisis de gestión administrativa. Editorial Panapo. Caracas, Venezuela

Sabino, A. (2007). Manual de conceptos básicos de gestión económico-financiera para personas emprendedoras. Editorial: BEAZ, S.A.U. México

Samuelson, P. y Nordhaus, W. (2005). Economía. Décimo séptima edición. Editorial McGraw-Hill. México

Skousen, M. y Addison, W. (2007). La economía en tela de juicio. Iberoamericana. Editorial McGraw-Hill. Colombia

Vera, M. (2001). Gestión financiera de la pequeña y mediana industria en la ciudad de Maracaibo". Revista de Ciencias Sociales (RCS). Vol. VII, No. 1. Venezuela (Pp. 178-199) 